illegality but of ineffective use of services by socially disadvantaged people and of services unsuited to their needs. Attitudes of the providers of services, real or perceived, are a further disincentive. Effective services can, however, be provided if the needs and wishes of prostitutes are acknowledged. ${ }^{1}$ Infection and violence are not confined to commercial sexual relationships, and changes in legislation would not guarantee impartial treatment by police.

Debate continues over the relative merits of decriminalisation and legalisation and, indeed, over whether specific effects might even be beneficial. Decriminalisation might seem the more limited reform, with legalisation a more radical option that would allow prostitutes to work in organised brothels as tax paying members of society. The English Collective of Prostitutes, however, argues that state controlled brothels would prove repressive with more, rather than less, exploitation of prostitutes. ${ }^{2}$ The collective also suggests that the motivation for changing the legal status of prostitution is more likely to result from concerns for the interests of those buying sexual services than from concerns for the interests of those selling them.

Prostitution will continue because a fall in demand is unlikely, but the level of prostitution might be reduced if the prostitute's need to sell sexual services was reduced. Providing alternative employment is difficult, and managing social problems, especially drug use and poverty, should be a prime objective. Prosecuting prostitutes does not reduce prostitution and in fact increases the prostitute's need to work to pay the fines imposed.

Although the precise benefits and best method of ending the illegality of prostitution may be debatable, this change is both necessary and desirable. Although the English Collective of Prostitutes believes that abolishing the relevant laws would abolish the stigma, social and moral attitudes rather than legal restraints are central to the problems of prostitution. Only when these are confronted can the problems of prostitution, legal or illegal, be resolved. Sadly, current trends in the social management of vulnerable people give little cause for optimism.

MARY HEPBURN

Royal Maternity Hospital,

Glasgow G4 0NA

Senior lecturer in women's reproductive health

1 Maclver N. Developing a service for prostitutes in Glasgow. In: Bury J, Morrison V, McLachlan S, C. Working with homen and AIDS. London: Tavistock/Routledge, 1992:85-95. 2 Lopez-Jones N. Legalising brothels. New Law fournal 1992; May 1:594-5.

\title{
How safe is tamoxifen?
}

\section{Only large randomised controlled trials can decide}

Tamoxifen has emerged as the main endocrine treatment for all stages of breast cancer. ${ }^{1}$ Adjuvant tamoxifen produces a survival advantage, irrespective of oestrogen receptor status, in postmenopausal women and a $40 \%$ decrease in second primary breast cancers overall. ${ }^{2}$ Associated physiological benefits of the oestrogen-like actions of tamoxifen include a fall in circulating cholesterol concentration, ${ }^{3}$ which could translate into fewer fatal myocardial infarctions ${ }^{4}$ or at least fewer admissions to hospital for cardiac conditions, ${ }^{5}$ and the maintenance of bone density in the lumbar spine. ${ }^{6}$ An estimated 4.5 million women years of experience with tamoxifen have now accrued, and treating all postmenopausal women with breast cancer with tamoxifen would save lives. ${ }^{7}$

Treating breast cancer once it has been diagnosed may not be enough, and pressures are being exerted for preventive strategies. Tamoxifen is an obvious choice because a sound laboratory rationale for its use exists ${ }^{8}$ and, for the foreseeable future, there is no prospect of an agent being developed that could provide its benefits in clinical practice. But how can the drug be evaluated?

Thirty years ago, believing that it might prevent some breast cancers, doctors might have prescribed tamoxifen to women at an increased risk, and this would have been acceptable. But times have changed, and randomised clinical trials-to protect doctors from bias and exaggerated claims by the pharmaceutical industry and patients from misguided doctors - are here to stay. They are modern medicine's most powerful tool, allowing the evaluation of efficacy and side effects without emotion.

The breast cancer prevention trial originating in the Royal Marsden Hospital continues to recruit volunteers at high risk of the disease. ${ }^{9}$ Already 2000 volunteers have been randomised, and recruitment continues despite concerns that tamoxifen causes liver tumours in rats. ${ }^{10}$ Meanwhile, the National Cancer Institute in the United States is sponsoring a prevention study with tamoxifen, and more than half of the required 16000 volunteers have been recruited. In Italy volunteers are also being randomised to a tamoxifen prevention trial that started this year; 20000 women will participate.

But fears have been raised about tamoxifen's safety. Most of the concern has focused on whether tamoxifen is a liver carcinogen. To the question "Is tamoxifen safe?" the answer must be, "Compared with what?" Tamoxifen promotes hepatic tumours in rats ${ }^{11}$ and causes the formation of adducts to DNA in rat livers. ${ }^{12}$ As oestrogens are notorious rat liver carcinogens and tamoxifen has oestrogenic properties in both rodents and humans, ${ }^{8}$ these effects are unsurprising.

A comparison with oral contraceptives is instructive here. Their oestrogen component induces tumours in rat liver, and women with at least five years' extended use are at increased risk of developing hepatocellular carcinoma. ${ }^{13}$ Objections currently being made to tamoxifen should therefore apply equally to oral contraceptives. But epidemiologists consider that the 10-fold increase in the risk of hepatocellular carcinoma with oral contraceptives is insignificant given the rarity of the tumour in Western countries ( 5 per 100000), and the convenience of avoiding pregnancy far exceeds the risk of liver cancer. In contrast, the incidence of hepatocellular carcinoma in patients treated with tamoxifen cannot be documented with any certainty: two cases have been reported in women receiving $40 \mathrm{mg} /$ day.

Reports of ocular and endometrial side effects of tamoxifen have been reviewed recently, ${ }^{14}$ but determining the true incidence of these effects outside published placebo controlled, randomised clinical trials is difficult. Women who have taken or are taking tamoxifen may develop endometrial carcinoma, but whether tamoxifen causes endometrial carcinoma or increases the rate of detection of occult disease is unknown. These risks can be quantified only in placebo controlled clinical trials.

One understandable concern is the expanded use of 
tamoxifen in younger women (aged 35) in some preventive trials. Information from epidemiological and laboratory studies suggests that the breast tissue is most receptive to carcinogens early in puberty ${ }^{15}{ }^{16}$ and antihormonal prevention is most effective nearer the time of the insult. ${ }^{17}$ This is why young women are being recruited. Only young women at the highest risk of developing breast cancer, however, are being included in the prevention studies.

Carefully monitored adjuvant clinical trials can guide the use of tamoxifen in normal women. The physiological impact of tamoxifen in premenopausal women warrants closer attention; the drug's effects on bone density and lipid concentrations might tip the balance of risks and benefits in its favour.

V CRAIG JORDAN

Robert H Lurie Cancer Center, Professor of cancer pharmacology

Northwestern University Medical School,

Chicago, IL 60611,

USA 1 Lerner LJ, Jordan VC. Development of antiestrogens and their use in breast cancer: eighth Cain
memorial award lecture. Cancer Res 1990;50:4177-89.
2 Early Breast Cancer Trials Collaborative Group. Systemic treatment of early breast cancer by
hormonal, cytotoxic and immune therapy: 133 randomized trials involving 33000 recoveries and
3 Love RR, Weibe DA, Newcomb PA, Cameron L, Leventhal H, Jordan VC, et al. Effects of tamoxifen on cardiovascular risk factors in postmenopausal women. Ann Intern Med 1991;115: $860-4$

4 McDonald CC, Stewart 1991;303:435-7.

. Rtqvist LE, Mattson A. Cardiac and thromboembolic morbidity among postmenopausal women with early-stage cancer in a randomized trial of adjuvant tamoxifen. $f$ Natl Cancer Inst 1993;85:1398-406.

6 Love RR, Mazess RB, Barden HS, Epstein S, Newcomb PA, Jordan VC, et al. Effects of tamoxifen on bone mineral density in postmenopausal women with breast cancer. $N$ Engl $f$ Med 1992;326:852-6.

7 Jordan VC. Can all postmenopausal women with a diagnosis of breast cancer receive benefit from tamoxifen? Reviews in Endocrine Related Cancer 1993;43:23-31.

8 Jordan VC. A current view of tamoxifen for the treatment and prevention of breast cancer. Fourteenth Gaddum memorial lecture. Br J Pharmacol 1993;110:507-17.

9 Powles TJ, Tillyer CR, Jones AL, Ashley SE, Treleaven J, Davey JB, et al. Prevention of breast cancer with tamoxifen-an update on the Royal Marsden Hospital pilot program. Eur 7 Cancer $1990 ; 26: 680-4$

10 Tamoxifen trial controversy. Lancet 1992;339:735.

11 Greaves P, Goonetillebe R, Nunn G, Topham J, Orton T. Two year carcinogenicity study of tamoxifen in Alderley Park Wistar-derived rats. Cancer Res 1993;53:3919-24.

12 White NH, DeMauts $F$, Davis A Smith $L L$ Crofton-Sleigh C Venitt $S$, 24 Genotoric potenga of tamoxifen and analogues in female Fischer F344n rats, DBAd and C578L6 mice and in human MCL-5 cells. Carcinogenesis 1992;13:2197-203.

13 Prentice RL. Epidemiologic data on exogenous hormones and hepatocellular carcinoma and selected other cancers. Prev Med 1991;20:38-46.

14 Morrow M, Jordan VC. The tamoxifen trial for breast cancer: clinical issues. In: DeVita VT, Hellman S, Rosenberg SA, eds. Cancer prevention. Philadelphia: Lippincott Healthcare, 1992:1-12.

15 Tokunaga M, Land CE, Yamamoto T, Asono M, Tokuoka S, Ezaki H, et al. Incidence of female breast cancer among atom bomb survivors, Hiroshima and Nagasaki 1950-1980. Radiat Res 1987;112:243-72.

16 Dao TL. Mammary cancer inductions by 7,12 dimethylbenzanthracene: relation to age. Science 1963;165:810-1.

17 Jordan VC, Morrow M. An appraisal of strategies to reduce the incidence of breast cancer. Stem Cells 1993;11:252-62.

\section{Long term management of patients after splenectomy}

\section{Pneumococcal vaccine, lifelong penicillin, and avoidance of protozoal infections}

For centuries it has been known that the spleen is not essential to life. But it represents about a quarter of the body's lymphoid tissue and, among other things, filters encapsulated organisms from the blood. Overwhelming infection may occur after removal of the spleen: the clinical syndrome comprises fulminant bacteraemia, disseminated intravascular coagulation, multiple organ failure, severe hypoglycaemia, and often rapid death. ${ }^{1}$ Its reported incidence after splenectomy varies from $0.9 \%$ to $69 \% \%^{23}$; mortality may exceed $50 \%$.

While the syndrome is most likely to occur within the first few years of surgery, especially in children and immunosuppressed patients, cases have occurred many years after splenectomy for trauma and other non-malignant conditions. ${ }^{56}$ Carrying out a proper randomised trial to decide how long prophylaxis should be given is impossible, but an analysis of published data suggests that it should be given to patients for the rest of their lives. We cannot agree with Holdsworth et al, who recently concluded that in people with no underlying pathology removal of the spleen was not associated with an increased risk of infection. ${ }^{2}$ Previously published studies, ${ }^{3}$ literature reviews, ${ }^{56}$ and a paper by Deodhar et al in this week's journal ( $\mathrm{p}$ 1408) ${ }^{7}$ support our contention that removing the spleen leads to an increased lifelong risk of infection. Deodhar et al report that postmortem review of patients who had had a splenectomy showed that $37 \%$ had died of infection, which in $11 \%$ of cases was pneumococcal. Nearly $60 \%$ of their patients who had had a splenectomy were not protected against infection. ${ }^{7}$

The commonest infecting organisms are Streptococcus pneumoniae (accounting for more than half the cases $^{8}$ ), Haemophilus influenzae, Neisseria meningitidis, and Escherichia coli. Although polyvalent pneumococcal vaccine prevents pneumococcal infection in immunocompetent patients who have not had a splenectomy, ${ }^{9}$ the antibody response to vaccination after splenectomy is impaired. ${ }^{10}$ The disease necessitating the splenectomy may also affect the antibody response to vaccination. Although currently available pneumococcal vaccine is effective against 23 strains of pneumococci, it does not protect against all pneumococcal infections. Despite this, vaccination is worth while. Current guidelines from the Department of Health recommend that pneumococcal vaccine should be given, if possible, two weeks before splenectomy and repeated every five to 10 years. ${ }^{11}$ Should vaccination prove impossible before surgery it should be carried out as soon as possible after. Kinnersley et al in this issue show that a large group of patients who have had a splenectomy remain unvaccinated ( $\mathrm{p} 1398) .{ }^{12}$

Prophylaxis with penicillin also protects against pneumococcal infection, although failures occur; some may be due to non-compliance. ${ }^{13-15} \mathrm{We}$ recommend that patients should take phenoxymethylpenicillin $250 \mathrm{mg}$ twice a day for life; patients allergic to penicillin should take erythromycin. Because episodes of overwhelming infection many years after splenectomy are rare, many patients may be unwilling to comply with long term prophylaxis. Most patients who develop overwhelming infection have a prodromal illness of at least a few hours: patients unwilling to take regular penicillin should be made aware of the risks and should have a readily available supply of penicillin so that they can start treatment immediately on developing suggestive symptoms. Wearing a Medic-Alert bracelet increases the awareness of patients and doctors alike.

Problems with penicillin resistant pneumococci may require us to review the recommendation. As $H$ influenzae is responsible for some cases of overwhelming infection ${ }^{16}$ vaccine against $H$ influenzae type $\mathrm{b}$ may be useful in patients after splenectomy and needs to be studied further.

In cases of trauma, salvage of the damaged spleen should be attempted when possible, especially in children. Conservative management of splenic injuries, with surveillance with ultrasonography and computed tomography, may avert 